\title{
RELAÇÃO ENTRE GASTOS COM SAÚDE,ÍNDICE DE ESFORÇOS DA ATENÇÃO BÁSICA EM SAÚDE E TIPOLOGIA DA ESTRUTURA DO SISTEMA DE SAÚDE DOS MUNICÍPIOS DO ESTADO DE SÃO PAULO
}

\begin{abstract}
RELATION BETWEEN HEALTH EXPENSES,BASIC ATTENTION IN HEALTH EFFORTS INDEX AND HEALTH SYSTEM STRUCTURE TYPOLOGY OF THE MUNICIPALITIES OF SÃO PAULO STATE
\end{abstract}

PATRÍCIA SIQUEIRA VARELA

Mestre e doutoranda em Controladoria e Contabilidade pela Faculdade de Economia,

Administração e Contabilidade (USP). Rua da Consolação, 930 - São Paulo - SP - CEP 01302-907 E-mail: psvarela@usp.br

MILTON CARLOS FARINA

Mestre em Administração pela Escola de Administração de Empresas de São Paulo (FGV) e doutorando em Administração pela Faculdade de Economia, Administração e Contabilidade (USP). Rua Ibipetuba, 130, Mooca - São Paulo - SP - CEP 03127-180 E-mail:milton_farina@uol.com.br 


\section{RESUMO}

Em um contexto marcado pela redemocratização do país e pelas propostas de reforma do Estado,a área da saúde tem se destacado pelos avanços no processo de descentralização,pela relevância dos dispêndios sociais munici pais nesta função e pela expansão dos sistemas de informações. Ao mesmo tempo,o atual modelo de gestão pública por resultados tem contribuído para o uso de indicadores sociais a fim de subsidiar o processo decisório,mas ainda existem o desafio de uma avaliação conjunta de indicadores de resultados/esforços e a aplicação de recursos orçamentários. O objetivo deste estudo foi apresentar uma análise descritiva a partir do contraste de relações entre categorias dos municípios paulistas em termos de gastos em saúde com recursos próprios por habitante,transferências do Sistema Único de Saúde (SUS) por habitante,ti pologia da estrutura do sistema de saúde e índice de esforço da atenção básica da saúde. Os dois últimos criados pela Fundação Sistema Estadual de Análise de Dados (Seade),órgão do governo do Estado de São Paulo. A análise do comportamento das quatro variáveis foi realizada por meio do teste qui-quadrado e da técnica de análise de correspondência. Obtiveram-se,como princi pais resultados,a associação entre os índices de esforço da atenção básica e os gastos em saúde com recursos próprios por habitante e o baixo poder de discriminação entre municípios pelos valores de transferências de recursos do SUS.

\section{PALAVRAS-CHAVE}

Setor saúde; Municípios; Infra-estrutura do Sistema de Saúde; Indicadoressociais; Gastos com saúde.

\section{ABSTRACT}

In a contextmarked by the re-democratization of the country and by the proposals of the State reform, the health area has stood outdue to the advances in the decentralization process, the relevance of the social expenditure of counties in 
this function, and the expansion of the information systems. Atthe same time, the currentmodel of public managementby results has contributed to the use of social indicators in order to subsidize the decision process, although there still is the challenge of a jointevaluation of indicators of results/efforts,and the application of budgeted resources. The objective of this studywas the presentation of a descriptive analysis based on the contrastof relationships among different categories of counties in the State of São Paulo,in terms of: expenditure in health with county-owned resources per inhabitant; transfers from Sistema Único de Saúde (SUS - governmental general health system) per inhabitant; typology of the health system structure,and ratio of basic attention to health effort. The last two items were created by Fundação Sistema Estadual de Análise de Dados (Seade - State System for Data Analysis Foundation),an agency belonging to the State of São Paulo. The analysis of the behavior of the four variables was carried outusing Q-square and the correspondence analysis technique,and the main results were: connection between the basic attention efforts index and the expenditures with health using county-owned resources per capita,and low discrimination power among counties in relation to values of transfer of SUS resources.

\section{KEYWORDS}

Health sector; Municipalities; Health System Infrastructure; Social indicators; Health expences.

\section{INTRODUÇÃO}

Nas últimas décadas,a administração pública brasileira tem vivido um intenso processo de transformação sob influência da redemocratização do país e da reforma do Estado.

Por um lado,a agenda democrática vem reivindicando: novos direitos sociais e políticos, eqüidade nos resultados das políticas públicas, descentralização do processo decisório,partici pação da sociedade civil na formulação,implementação e controle dos programas governamentais,valorização das instituições representativas, accountability e transparência (SCHOMMER,2003,p. I05).

Por outro,a adoção de idéias liberais,para combater a crise dos últimos tempos,colocou em questionamento o papel do Estado como o único responsável pela solução dos problemas sociais e do desenvolvimento econômico,incentivando as privatizações, as terceirizações, as transferências da execução dos serviços não-exclusivos para o setor público não-estatal,a desregulamentação,o ajuste fiscal,a eficiência na aplicação dos recursos públicos e a gestão voltada para o cidadão (BRESSER PEREIRA,I998,p. 60). 
Apesar dos propósitos diferentes,o movimento de descentralização tem sido uma estratégia utilizada tanto pelo processo de redemocratização quanto pela reforma do Estado,favorecendo a transferência de poder,recursos e atribuições para os governos locais. O município é o ente federativo que mais tem sofrido os impactos desse processo de mudança da administração pública brasileira,por ser considerado depositário das reais possibilidades de intervenção do Estado e, também,por estar despreparado para assumir as novas responsabilidades a ele direcionadas.

A mudança do modelo de gestão do setor público ocasionou uma ênfase nos aspectos de planejamento e controle,visando à utilização ótima dos recursos disponíveis. Ao mesmo tempo,o maior acesso a fontes de informações pela população tem favorecido um maior controle social.

Observam-se,nesse contexto,o redesenho de alguns instrumentos e ferramentas de gestão e a incorporação de outros,como é o caso da expansão das bases de dados e do uso de indicadores sociais para subsidiar os gestores públicos no processo decisório.

Os indicadores sociais "[...] passaram a integrar o vocabulário corrente dos agentes políticos responsáveis,em última instância,pela definição das prioridades das políticas sociais e a locação dos recursos públicos" (JANNUZZI,200I,p. II).

A área da saúde tem sido foco de diversos estudos na atualidade,pois representa uma das políticas públicas em que o processo de descentralização mais tem avançado,com a implantação do Sistema Único de Saúde (SUS),além da relevância dos dispêndios sociais munici pais nessa função e o crescimento dos sistemas de informações em saúde.

Existem informações disponíveis sobre diversos aspectos da saúde,inclusive em forma de indicadores, como nascidos vivos, mortalidade, produção, perfil da população, saúde materna, orçamentos públicos em saúde etc. Porém,são apresentadas de forma dissociada,o que dificulta a avaliação da performance dos municípios.

Uma das princi pais preocupações relacionadas à gestão pública é como alocar os escassos recursos públicos na prestação de serviços de maneira eficiente, eficaz e efetiva. Portanto,o interesse não se restringe apenas às modificações no estado de saúde da população,mas também como os recursos foram utilizados para alcançar tais modificações.

A dificuldade de estabelecer relacionamentos entre aplicação de recursos públicos e resultados dos programas sociais municipais faz parte de uma discussão mais ampla que é estabelecer relações entre indicadores de processos e indicadores de produtos/resultados.

Os resultados dos programas relacionam-se com o grau de alcance dos objetivos traçados com base na realidade empírica que se pretendia modificar, 
por exemplo,diminuição da mortalidade infantil. Para avaliar um programa governamental,é preciso tentar determinar até que ponto ele contribuiu para as modificações verificadas na situação de saúde da população em um determinado período de tempo,ou seja,se existiu efetividade. Além disso,é relevante averiguar se o que foi proposto fazer foi realizado (eficácia),por exemplo,o número de consultas pediátricas (produtos das ações de saúde),e se isso ocorreu com o mínimo de recursos possíveis,sem sacrificar a qualidade (eficiência).

No processo de avaliação das ações governamentais,existem limitações em relacionar diretamente recursos públicos usados e resultados alcançados. De acordo com Aristigueta etal. (2001,p. 262), não é possível afirmar que, se o ente governamental é eficiente ou eficaz,necessariamente haverá mudanças nas condições de saúde e bem-estar da população,mas, conforme Burkhead (I97I,p. I86),informações sobre o desempenho (eficiência e eficácia) poderão contribuir para o julgamento de valor,em termos políticos e feito para a sociedade,sobre o alcance dos programas sociais.

A avaliação do desempenho dos governos,por sua vez,não pode prescindir de uma comparação entre os serviços de saúde prestados e os recursos usados para tal,a fim de melhorar as ações governamentais relativamente a seus custos e à sua forma de financiamento. Assim,este estudo teve como objetivo fazer uma análise descritiva a partir do contraste de relações entre categorias dos municípios,nos seguintes aspectos: tipologia da estrutura do sistema de saúde,índice de esforços da atenção básica da saúde,gastos em saúde com recursos próprios por habitante e transferências do SUS por habitante.

Para isso,primeiro avaliou-se a associação entre as variáveis pelo teste quiquadrado e estudaram-se as relações entre todas as categorias das quatro variáveis em uma análise de correspondência,técnica estatística multivariada que permite que o pesquisador visualize,em um mapa perceptual,as proximidades/ distâncias entre as categorias das variáveis, indicando relações existentes entre elas (HAIR JUNIOR etal.,I998,p. 548).

Existem poucos estudos que buscam conciliar recursos orçamentários e produtos/resultados das políticas públicas e,normalmente,usam técnicas de otimização baseadas em programação linear,como: Afonso etal. (2003), Sousa etal. (2003), Borger e Kerstens (I996),Borger etal. (I994), Gupta e Verhoeven (200I) e Afonso e Aubyn (2004). Destaque também deve ser dado ao trabalho de Scarpin (2006) que usou a análise de regressão múlti pla para estimar um modelo de previsão dos valores futuros do Índice de Desenvolvimento Humano Municipal (IDH-M).

Este estudo torna-se relevante para possibilitar uma avaliação conjunta dos indicadores de estrutura e esforços e dos gastos públicos em saúde dos muni- 
cípios paulistas,com inovação quanto à abordagem de comparação dos dados. Apesar de a análise de correspondência não ser tão robusta quanto às técnicas usadas pelos outros estudos, permite visualizações rápidas das relações entre variáveis,sendo uma boa opção à exploração de informações tanto por pesquisadores quanto por gestores públicos e cidadãos.

\section{ÍNDICE DE ESFORÇO EM SAÚDE DA FUNDAÇÃO SEADE}

No setor público,podem ser observadas diversas iniciativas e experiências para o uso de indicadores sociais. A mais conhecida delas é a da Organização das Nações Unidas (ONU) que,na década de I990,criou o Índice de Desenvolvimento Humano (IDH), apresentando uma inovação ao introduzir,em sua concepção,as variáveis longevidade e educação,além da renda,para comparar o grau de desenvolvimento dos países.

Outras experiências surgiram a partir do IDH,como o Índice Paulista de Responsabilidade Social (IPRS) que construído pela Fundação Sistema Estadual de Análise de Dados (Seade),órgão do governo do Estado de São Paulo,com o objetivo de classificar os municípios paulistas segundo a qualidade de vida dos seus habitantes. Para o cálculo do IDH, são utilizados três índices, aos quais são atribuídos pesos iguais: longevidade (esperança de vida ao nascer),educação (número médio de anos de estudo e taxa de analfabetismo) e renda (renda familiar per capita média ajustada).

O IPRS tem como objetivo a classificação dos municípios do Estado de São Paulo segundo a qualidade de vida dos seus habitantes. Para isso,foram abordadas as três dimensões abrangidas pelo IDH (renda,longevidade e escolaridade), mas utilizando-se outras variáveis mais condizentes com a realidade munici pal. Houve também a preocupação em adotar procedimentos metodológicos para o desenvolvimento de um conjunto de indicadores sensível a variações no curto prazo,favorecendo a avaliação periódica da gestão pública.

$\mathrm{Na}$ definição operacional do IPRS,consideraram-se indicadores obtidos de dados secundários provenientes de registros administrativos, geralmente de atualização anual,que possibilitassem análises e interpretações por município. Além disso,para mensurar e analisar o processo de desenvolvimento dos municípios do Estado de São Paulo,desde o início do projeto,buscou-se a elaboração de indicadores de resultados e esforços.

Uma proposta mais consistente de elaboração de indicadores de esforços é apresentada no relatório "Geração de indicador de esforços em saúde" encami- 
nhado à Assembléia Legislativa do Estado de São Paulo junto com o relatório sobre as atua lizações,avaliações e aprimoramento do IPRS 2002. É um desdobramento da proposta metodológica do índice,resultando na

[...] definição operacional de novas medidas de esforços em saúde que possibilitam apreender e,de certa forma,antecipar o futuro de determinadas ações e serviços públicos de promoção,proteção e recuperação da saúde atualmente em implementação (FUNDAÇÃO SEADE,2003,p. 4).

As duas dimensões abrangidas pelo estudo são: índice de esforço em atenção básica à saúde e ti pologia da estrutura do sistema de saúde.

\section{1 ÍNDICE DE ESFORÇO EM ATENÇÃO BÁSICA À SAÚDE}

De acordo com a Fundação Seade (2003,p. I8):

Este índice mede a disponibilidade e a produtividade dos recursos humanos mobilizados pelas prefeituras para a oferta de amplo conjunto de procedimentos médico-sanitários, de natureza predominantemente preventiva,cuja implementação é uma das prioridades do Ministério da Saúde para o processo de municipalização do setor.

Para a definição operacional do índice,foram empregados indicadores referentes a duas dimensões: a disponibilidade de recursos humanos para a produção de serviços de atenção básica à saúde por habitante e a produção de serviços por estes recursos por habitante.

Com base na definição operacional,adotaram-se dois indicadores para a quantificação dos recursos humanos qualificados e potencialmente disponíveis nas prefeituras: I. médicos por habitante e 2 . médicos em especialidades por habitante. Já a produção de serviços foi medida por três indicadores: a) consultas médicas em especialidades por habitante; b) consultas médicas em pediatria por habitante na faixa etária entre o e I4 anos; e c) consultas médicas em ginecoobstetrícia por habitante do sexo feminino,na faixa etária entre I5e 39 anos. Os pesos de cada uma das variáveis encontram-se na Figura I. 


\section{FIGURA I}

\section{DESCRIÇÃO DO ÍNDICE DE ESFORÇOS EM ATENÇÃO BÁSICA A SAÚDE}

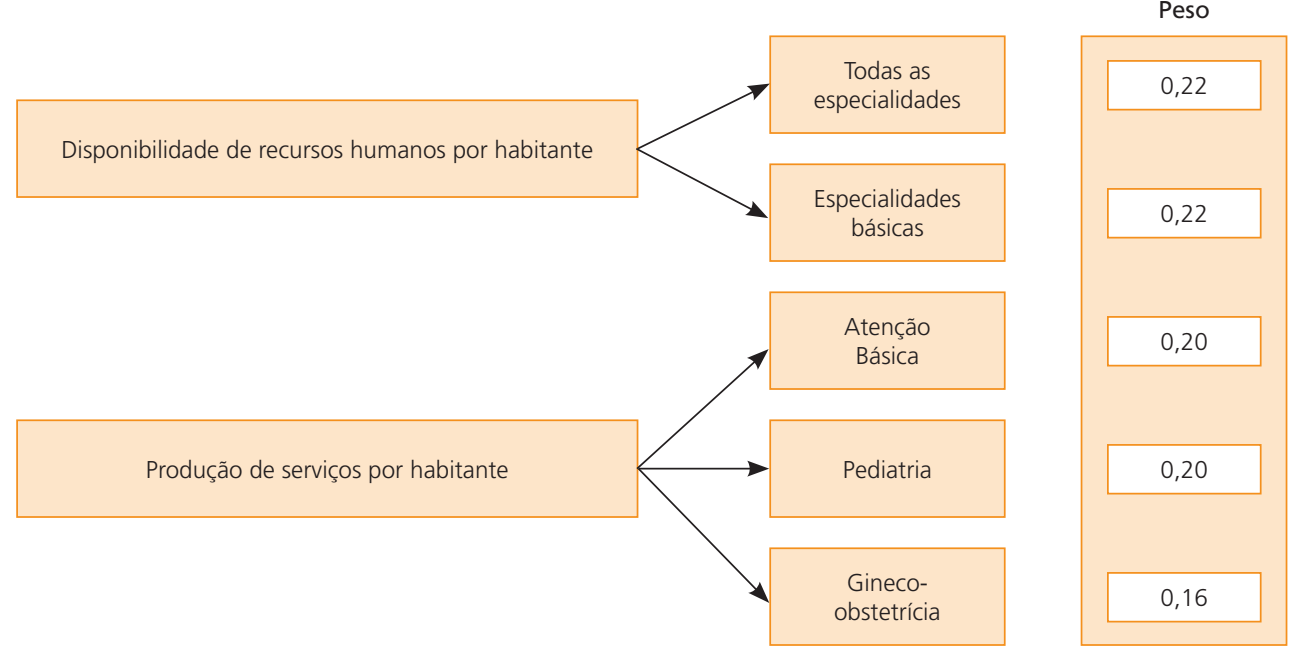

Fonte: Fundação Seade (2003,p. II).

Todos os indicadores foram condensados em uma medida única correspondente à média ponderada dos valores observados em cada município,conforme os pesos atribuídos a cada um deles. Os municípios paulistas foram classificados em escala intervalar entre o e ıoo,traduzindo a capacidade de mobilização de recursos para a organização do modelo de atenção à saúde no âmbito do SUS.

\subsection{TIPOLOGIA DA ESTRUTURA DO SISTEMA DE SAÚDE}

O objetivo da ti pologia é diferenciar os estabelecimentos de saúde em atividade nos distintos municípios do Estado de São Paulo,conforme a capacidade instalada para internação de pacientes, a vinculação ao SUS e a complexidade tecnológica da respectiva produção ambulatorial (FUNDAÇÃO SEADE,2003, p. I8). Os municípios foram classificados em cinco categorias definidas com base em características investigadas pela Pesquisa de Assistência Médico-Sanitária (AMS) da Fundação Instituto Brasileiro de Geografia e Estatística (IBGE),em I999,e dados da produção ambulatorial do SUS,em 200I,ordenados segundo o grau de complexidade tecnológica. As categorias são: sem-internação,baixa complexidade,média complexidade,alta complexidade e município de São Paulo.

Os cincos ti pos diferenciam-se,fundamentalmente,pela capacidade de internação e pela complexidade tecnológica da produção ambulatorial. Esta última, 
por sua vez,tem relação direta com a vinculação dos estabelecimentos de saúde ao SUS. A capacidade de internação diferencia o primeiro grupo dos demais,e a complexidade tecnológica é o principal fator de diferença entre o segundo,o terceiro e o quarto tipos. O município de São Paulo constituiu,isoladamente,o quinto grupo por sua peculiar concentração de procedimentos ambulatoriais de média e alta complexidade. Sendo assim,consideram-se o primeiro e o quinto grupo,incomparáveis entre si,e com os demais grupos (FUNDAÇÃO SEADE, 2003,p. I8).

Essa segunda dimensão do indicador de esforços em saúde surgiu da necessidade de evitar comparações entre municípios com estruturas muito distintas de atendimento à saúde,o que poderia levar a conclusões inadequadas. Os indicadores de esforços em atenção básica,analisados separadamente, tendem

[...] a favorecer de maneira enganosa os municípios menores,uma vez que tais ações compõem praticamente todos os seus esforços na área.

$[\ldots]$

Os procedimentos mais complexos, sobretudo nos municípios maiores que devem consumir boa parte de seus esforços -,não são incorporados a esse conjunto de indicadores, que tampouco consideram a presença de instituições privadas na área da saúde,particularmente relevantes nesse estrato de municípios (FUNDAÇÃO SEADE,2003,p. I3).

A Fundação Seade ainda reconhece a importância do Sistema de Informações sobre Orçamentos Públicos em Saúde (Siops) como gerador de informações relevantes para a mensuração dos esforços públicos munici pais em saúde. Em um dos anexos do relatório "Geração de indicador de esforços em saúde",procurou estabelecer a relação entre dados financeiros e orçamentários referentes ao ano de 200 I e a tipologia da estrutura do sistema de saúde.

\section{FINANCIAMENTO DO SETOR MUNICIPAL DE SAÚDE}

Segundo os artigos I95e I9 8 da Constituição Federal de I988,o financiamento do Sistema Único de Saúde é uma responsabilidade comum da União, dos Estados,do Distrito Federal e dos municípios. As fontes de financiamento do setor público municipal de saúde são basicamente: recursos oriundos das transferências do Fundo Nacional de Saúde e recursos orçamentários próprios. 
A Emenda Constitucional n. 29/200o determinou a vinculação e estabeleceu a base de cálculo e os porcentuais mínimos de recursos orçamentários que cada um dos entes da Federação estariam obrigados a aplicar em ações e serviços de saúde.

A maior parte dos recursos aplicados pela União financia a prestação de serviços pelos municípios na área da saúde,o que representa uma fonte expressiva de recursos para estes,já que a forma de distribuição dos recursos depende da condição de gestão do município.

As condições de gestão significam "[...] a declaração dos compromissos assumidos por parte do gestor perante os outros gestores e perante a população sob sua responsabilidade" (BRASIL,2003b). A partir da publicação da Norma Operacional Básica do SUS (NOB/SUS or/96),os municípios poderiam habilitar-se em duas condições: gestão plena da atenção básica ou gestão plena do sistema munici pal. A diferença entre essas condições de gestão é devida aos níveis de complexidade dos serviços de competência do município.

Os municípios em gestão plena da atenção básica recebem transferência, regular e automática,dos recursos referentes ao Piso da Atenção Básica Ampliado (PAB-A),correspondente ao financiamento do elenco de procedimentos básicos e do incentivo de vigilância sanitária (BRASIL,2003b).

O PAB-A é composto de uma parte fixa de recursos (PAB-A fixo) destinados à assistência básica e de uma parte variável (PAB-A variável) relativa a incentivos para o desenvolvimento dos programas executados nesse nível de atenção,como: Programa Saúde da Família (PSF),Programa de Agentes Comunitários de Saúde (Pacs),Assistência Farmacêutica,Vigilância Sanitária e Combate às Carências Nutricionais. Todos os recursos são transferidos direta e automaticamente do Fundo Nacional de Saúde para os fundos munici pais correspondentes. Os recursos correspondentes à parte fixa são obtidos pela multiplicação de um valor per capita nacional pela população do município.

O Ministério da Saúde também tem políticas de indução para ampliação da oferta de serviços em áreas estratégicas,cujo financiamento é feito pelo Fundo de Ações Estratégicas e Compensação (Faec). Os municípios recebem,complementarmente a seus tetos financeiros,os recursos necessários para custeio dos procedimentos relacionados a essas ações estratégicas.

Para os municípios em gestão plena do sistema munici pal,além dos recursos do PAB-A,também existem transferências fundo a fundo de recursos relacionados aos procedimentos de média e alta complexidade,conforme a possibilidade de prestação de serviços pelos mesmos.

A Norma Operacional de Assistência à Saúde (NOAS/SUS OI/O2) define como prerrogativas dos municípios em gestão plena do sistema municipal a transferência,regular e automática,dos recursos referentes aos valores per capita 
para o financiamento dos procedimentos da média complexidade I,após qualificação da microrregião na qual estão inseridos,para sua própria população e,caso seja módulo assistencial,para sua população e a população de outros municípios que compõem a microrregião.

Além dos recursos de transferência do SUS,os fundos munici pais de saúde também recebem aportes dos orçamentos dos próprios municípios, de acordo com os limites determinados pela Emenda Constitucional n. 29/2000.

Para os municípios,os porcentuais de vinculação representam $15 \%$ das suas receitas de impostos e transferências recebidas. Existia a previsão do prazo de cinco anos para que os porcentuais de recursos a serem destinados para financiamento do setor pelos governos subnacionais fossem alcançados. Os municípios que estivessem aplicando porcentuais inferiores ao fixado deveriam elevá-los, gradualmente,até o exercício financeiro de 2004,reduzindo a diferença à razão de pelo menos um quinto por ano,com aplicação mínima de $7 \%$ a partir de 2000.

De acordo com Mendes e Marques (2003,p. 393), a descentralização da política de saúde para os municípios deve ser entendida de forma relativa,pois os recursos federais compõem a maior parte do financiamento do sistema,aproximadamente $54,6 \% \mathrm{em} 200 \mathrm{I}$. Os critérios de transferência de parte significativa de tais recursos relacionam-se a ações preconcebidas pelo Ministério da Saúde, restringindo a autonomia da gestão munici pal quanto à aplicação dos recursos conforme a necessidade de saúde da população pela qual é responsável. Além disso,como afirma Barros (2003,p. 42),o processo de descentralização no setor saúde apresenta a clara característica de "[...] fortalecimento da presença dos municípios na atenção básica e a restrição ao processo de assunção da responsabilidade plena pela gestão sanitária".

Arretche (2003,p. 33I) argumenta que os governos locais assumiram a gestão da atenção básica à saúde,contudo não há garantias intrínsecas à gestão municipal que promova responsabilidade,eficiência,acesso universal e eqüidade: "A qualidade da ação dos governos depende em grande medida dos incentivos e dos controles a que estão submetidos". Além do poder do Ministério da Saúde de indução sobre as decisões dos governos locais,Arretche (2003,p. 343) destaca a existência de instrumentos de controle e fiscalização disponíveis para eleitores, representantes dos usuários e da sociedade civil.

\section{PROCEDIMENTOS METODOLÓGICOS}

As variáveis utilizadas no estudo são provenientes de fontes de dados secundários. O índice de esforço em atenção básica à saúde e a ti pologia da estrutu- 
ra do sistema de saúde foram retirados do relatório "Geração de indicador de esforços em saúde” (FUNDAÇÃO SEADE,2003). Já os gastos em saúde com recursos próprios por habitante e transferências do SUS por habitante foram extraídos do Sistema de Informações em Orçamentos Públicos (Siops),ano-base 200I (DATASUS, 2004).

Para verificar as relações entre as variáveis sob estudo,foi aplicada a análise de correspondência,técnica estatística multivariada que estabelece a associação entre categorias de características descritivas ou atributos específicos de objetos ou casos,por exemplo,municípios (HAIR JUNIOR etal.,I998,p. 548).

De acordo com Hoffman e Franke (I986,p. 2I3),a análise de correspondência objetiva o agrupamento das categorias (de linhas e colunas) de respostas de duas ou mais variáveis. A partir da decomposição de uma matriz de distâncias de qui-quadrado,o algoritmo da análise de correspondência produz uma série de matrizes que são aplicadas às linhas e colunas de uma tabela de contingência para que sejam produzidas distâncias entre os pontos,base para geração de um gráfico em dimensões reduzidas.

Antes de processar a análise de correspondência,a realização do teste quiquadrado é recomendada para esclarecer se a distribuição das variáveis por categoria é a leatória ou se há um padrão determinado por interdependência entre as variáveis. Conforme Maroco (2003,p. 86), o teste qui-quadrado serve para testar se a freqüência com que os elementos da amostra se repartem pelas classes de uma variável categorizada é ou não idêntica.

Com a aplicação da análise de correspondência,buscou-se saber se a política de financiamento (recursos próprios e transferências do SUS) guardava relação com a ti pologia da estrutura do sistema de saúde e o índice de esforços em atenção básica em saúde.

Com base nos dados do Siops e do IPRS,foram definidas as seguintes variáveis:

- DPSHAB: despesa do município com saúde por habitante;

- TSUSHAB: transferência de recursos SUS ao município por habitante;

- Índices: índice de esforço em atenção básica à saúde;

- Tipologia: ti pologia da estrutura do sistema de saúde.

A aplicabilidade da análise de correspondência refere-se à necessidade de tentar identificar algum vínculo de associação entre as categorias das variáveis sob estudo. A única variável originalmente em escala ordinal era a tipologia,as outras três variáveis estavam em escala intervalar,portanto foi preciso que passassem por um processo de categorização. Para tanto,utilizou-se como critério a divisão pelos quartis das variáveis. 
Os municípios foram ordenados segundo o valor de cada uma das variáveis, separadamente,e classificados em quatro grupos: $25 \%$ dos valores inferiores ao primeiro quartil, $50 \%$ inferiores ao segundo quartil, $75 \%$ inferiores ao terceiro quartil e $25 \%$ superiores ao terceiro quartil. Assim,por exemplo,a variável DPSHAB foi transformada em QDPSHAB,em que o valor I indica os municípios com gastos situados em até $25 \%$,o valor 2 entre $25 \%$ e $50 \%$,o valor 3 entre 50\% $75 \%$ e o valor 4 acima de $75 \%$.

A Tabela I indica os quartis que definem as quatro categorias de cada variável.

\section{TABELA I}

QUARTIS DAS VARIÁVEIS: DESPESA DO MUNICÍPIO COM SAÚDE

POR HABITANTE,TRANSFERÊNCIAS DE RECURSOS SUS

POR HABITANTE E ÍNDICE DE ESFORÇO EM ATENÇÃO

BÁSICA A SAÚDE

\begin{tabular}{cccc}
\hline QUARTIL & DPSHAB & TSUSHAB & INDICES \\
\hline Primeiro & 66,1900 & 17,5725 & 18,0000 \\
\hline Segundo & 88,2500 & 31,2550 & 27,0000 \\
\hline Terceiro & 127,2675 & 47,9300 & 38,0000 \\
\hline
\end{tabular}

A análise foi realizada em relação a 640 dos 645 municípios do Estado de São Paulo. O município de São Paulo foi retirado do banco de dados por ser o único classificado com valor 5 na variável ti pologia,podendo ser considerado um outlier. Os outros quatro municípios desconsiderados no estudo não apresentavam valor de pelo menos uma das variáveis,e a decisão foi a eliminação de tais casos.

\section{ANÁLISE dOS RESULTADOS}

Inicialmente,foi realizado o teste qui-quadrado das variáveis QDPSHAB, QTSUSHAB e QINDICES com a variável ti pologia,a fim de se ter uma avaliação prévia da pertinência do uso da análise de correspondência. As hi póteses testadas foram:

- Ho: Não existem diferenças relativamente à freqüência esperada de cada uma das categorias das outras variáveis nas categorias da variável ti pologia.

- Hi: Existem diferenças significativas quanto à freqüência esperada de cada uma das categorias das outras variáveis nas categorias da variável ti pologia. 
O teste do qui-quadrado,representado na Tabela 2,apresenta um nível de significância de 0,000 que é inferior a 0,05 (nível de significância do teste), levando à rejeição de Ho: independência entre as variáveis: ti pologia e QDPSHAB,TIPOLOGIA e QTSUSHAB,TIPOLOGIA e QÍNDICES. O teste indica associação entre a estrutura do sistema de saúde com o montante da despesa própria com saúde por habitante,o montante de recursos recebidos por transferência do SUS por habitante e o índice de esforço em atenção básica.

TABELA 2

RESULTADOS DO TESTE QUI-QUADRADO ENT'RE AS VARIÁVEIS:

TIPOLOGIA DA ESTRUTURA DO SISTEMA DE SAÚDE COM

DESPESA DO MUNICÍPIO COM SAÚDE POR HABITANTE,

TRANSFERÊNCIA DE RECURSOS SUS POR HABITANTE E ÍNDICE DE ESFORÇO EM ATENÇÃO BÁSICA A SAÚDE

\begin{tabular}{lcc}
\hline & VALOR DA ESTATISTICA & PROBABILIDADE \\
\hline QDPSHAB & 134,747 & 0,000 \\
\hline QTSUSHAB & 37,336 & 0,000 \\
\hline QÍNDICES & 137,059 & 0,000 \\
\hline
\end{tabular}

Considerando os resultados favoráveis do teste qui-quadrado,foi realizada a análise de correspondência múlti pla HOMALS ou análise de homogeneidade via mínimos quadrados alternados. De acordo com Pestana e Gageiro ([I99-],p. 368), tal técnica "[...] transforma os dados qua litativos associando-lhes quantificações óptimas (scores) que permitem não só a sua representação gráfica,bem como a maior separação entre as categorias".

As quatro variáveis sob estudo foram representadas em duas dimensões, com o intuito de identificar se existe correspondência entre a ti pologia da estrutura do sistema de saúde,os quartis do índice de esforço da atenção básica,os quartis dos recursos próprios aplicados em saúde por habitante e os quartis das transferências dos SUS por habitante.

A expectativa era de quanto maiores os índices de esforço da atenção básica à saúde, maiores os recursos aplicados em saúde, tanto os próprios como os recebidos por transferência. Em relação a estes últimos,esse fato deveria acontecer porque os critérios de distribuição de parte significativa de tais recursos relacionam-se a ações preconcebidas pelo Ministério da Saúde como forma de priorização da atenção básica. 
Já em relação à ti pologia,a expectativa era de quanto maior a complexidade da estrutura do atendimento à saúde,maior o valor de recursos próprios investidos por habitante,uma vez que os procedimentos de média e alta complexidade, em grande parte curativos,possuem custo maior se comparado com os procedimentos da atenção básica. Também porque as transferências do SUS são insuficientes para cobrir os custos dos procedimentos previstos para atendimento da população sob responsabilidade dos municípios.

\subsection{ANÁLISE DOS EIGENVALUES E DAS MEDIDAS DE DISCRIMINAÇÃO}

Os valores próprios (eigenvalues) informam a contribuição de cada uma das dimensões para explicar a variabilidade contida nos dados e constituem os valores médios das medidas de discriminação das variáveis em relação a cada dimensão. Os valores próprios variam entre o e I,ocorrendo o I quando as categorias que caracterizam cada dimensão são por ela explicadas na totalidade (PESTANA; GAGEIRO,[I99-],p. 37I). Neste estudo,os eigenvalues da dimensão I é o,4884 e da dimensão 2 é 0,3179,conforme os dados da Tabela 3.

TABELA 3

VALORES EIGENVALUES PARA CADA DIMENSÃO

DIMENSÃO

1

2
EIGENVALUE

0,4884

0,3179

As medidas de discriminação informam as variáveis que mais contribuem para a definição de cada uma das dimensões. As medidas de discriminação variam entre $\mathrm{O}$ e I,assumindo valor I quando a discriminação é perfeita e os objetos pertencem exclusivamente a um dos grupos. De acordo com os dados da Tabela 4,as variáveis QDPSHAB e QINDICES contribuem para a discriminação da dimensão I. A variável QTSUSHAB contribui para a discriminação da dimensão 2. Já a variável ti pologia contribui razoavelmente para a discriminação tanto da dimensão I quanto da dimensão 2. 
TABELA 4

PODER DE DISCRIMINAÇÃ O DAS VARIÁVEIS

\begin{tabular}{ccc}
\hline VARIÁVEL & \multicolumn{2}{c}{ DIMENSÃO } \\
\cline { 2 - 3 } & 1 & 2 \\
\hline TIPOLOGIA & 0,602 & 0,470 \\
\hline QDPSHAB & 0,644 & 0,177 \\
\hline QTSUSHAB & 0,050 & 0,489 \\
\hline QINDICES & 0,658 & 0,136 \\
\hline
\end{tabular}

Assim,a primeira dimensão apresenta a ti pologia da estrutura do sistema de saúde em relação à despesa própria do município com saúde por habitante e ao índice de esforço da atenção básica à saúde. Já a segunda dimensão,menos discriminativa,revela a associação entre tipologia da estrutura e transferências de recursos do SUS por habitante.

\section{FIGURA 2}

MAPA PERCEPTUAL

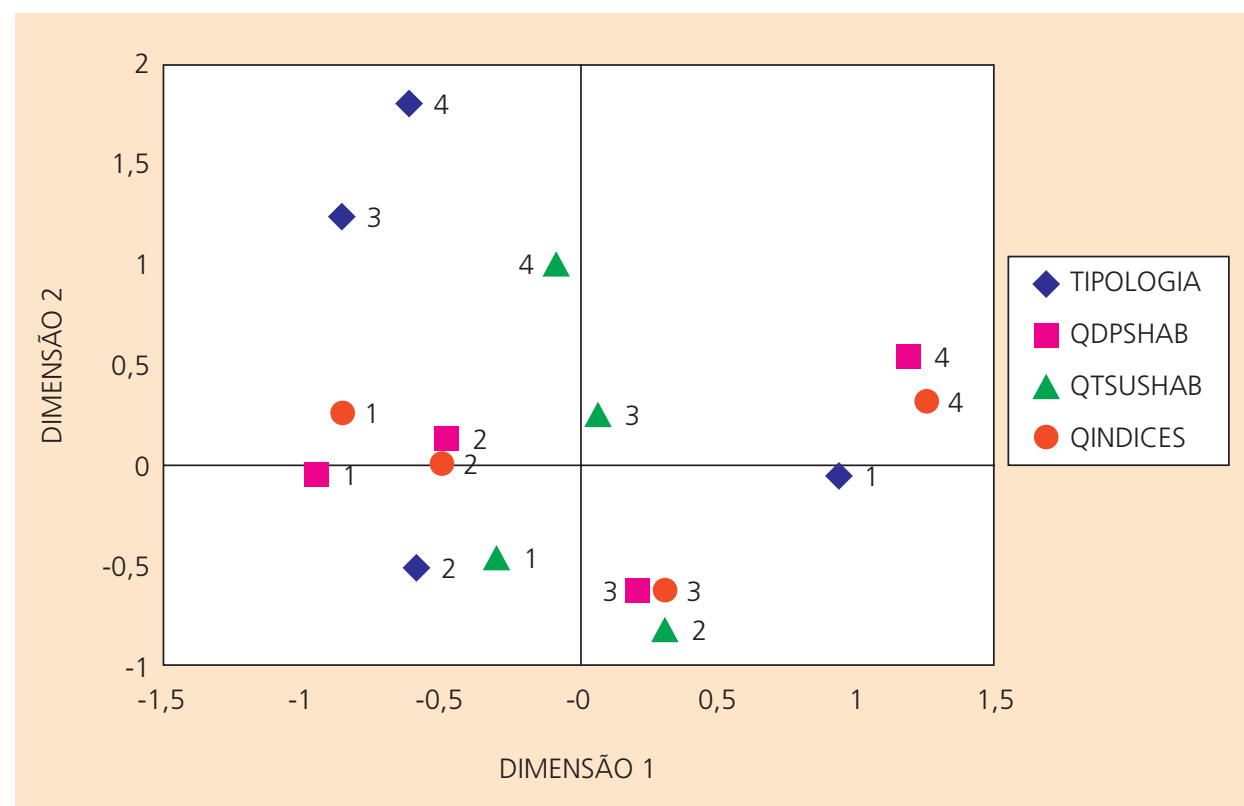




\subsection{ANÁLISE DO MAPA PERCEPTUAL}

Pelo mapeamento (Figura 2), percebe-se nitidamente a correspondência entre as categorias I,2,3 e 4 das variáveis QDPSHAB e QINDICES, indicando que quanto maior a despesa com recursos próprios por habitante,maior o índice de esforços em atenção básica à saúde.

Observa-se também a associação da ti pologia I (municípios sem internação) com o quarto quartil das variáveis QDPSHAB e QINDICES. Se,por um lado,corrobora a tendência de o índice de esforço em atenção básica favorecer os municípios menores, por outro,indica que os recursos próprios aplicados,em termos per capita,são maiores nesses municípios. Para as outras ti pologias,não é possível perceber essa mesma relação com as outras categorias das duas variáveis.

O segundo quartil da variável QTSUSHAB com o terceiro quartil das variáveis QDPSHAB e QINDICES forma um outro grupo. As outras categorias das três variáveis não estão visivelmente associadas. Reforça-se,assim,a idéia de que o desempenho dos municípios na atenção básica está mais fortemente relacionado com o dispêndio de recursos próprios na função saúde.

A variável QTSUSHAB é a que menos contribui para a discriminação dos municípios,por apresentar valores mais próximos da origem. Apesar disso,percebe-se uma certa tendência de associação com as categorias da ti pologia: primeiro quartil com a ti pologia 2 ,e quarto quartil com a ti pologia 3 .

De modo geral,a ti pologia 3 (média complexidade) e a ti pologia 4 (alta complexidade) estão distantes das categorias das outras variáveis.

\section{CONSIDERAÇÕES FINAIS}

A Fundação Seade criou as ti pologias da estrutura do sistema de saúde para propiciar a avaliação adequada dos municípios,a fim de evitar comparações dos índices de esforços em atenção básica de estruturas muito distintas de atendimento à saúde,pois haveria a tendência de favorecer de maneira enganosa os municípios menores,uma vez que tais ações representam todos os seus esforços.

Com exceção dos municípios classificados na ti pologia I (sem internação),a associação entre o índice de esforços em atenção básica e a ti pologia da estrutura do sistema de saúde não ficou nítida. Portanto,as ti pologias 2,3 e 4 (baixa complexidade,média complexidade e alta complexidade,respectivamente) parecem não resolver o problema de evitar comparações de municípios muito diferentes.

Além disso,quando se agregaram à análise dados orçamentários e financeiros,ficou claro que os municípios com maior índice de esforço em atenção 
básica são aqueles que apresentaram um maior valor de despesa com recursos próprios por habitante e que correspondiam exatamente aos municípios classificados na ti pologia I,ou seja,municípios com estruturas mais simples de atendimento à saúde,indicando que estes investem fortemente nas ações de promoção e prevenção da saúde.

Assim,inicialmente, a descrição contraria as conclusões de outros estudos que indicam a forte influência do governo federal nas decisões dos governos locais. Parece que os municípios agem com certa autonomia quanto à alocação dos recursos próprios, talvez a indução das decisões venha de outros atores sociais. Uma outra possibilidade é a não-adequação do índice de esforços da atenção básica formulado pela Fundação Seade.

A expectativa de quanto maior for a complexidade da estrutura do atendimento à saúde maior será o valor de recursos próprios investidos por habitante não se configura no mapa perceptual,ao contrário,as categorias que representam os maiores valores de despesas com recursos próprios estão mais próximas das ti pologias i e 2 .

O distanciamento das ti pologias 3 (média complexidade) e 4 (alta complexidade) das categorias das outras variáveis sugere a utilização de outros dados, como gastos com plano de saúde,para entender melhor a política de financiamento dos serviços de saúde prestados pelos municípios classificados em tais tipologias,uma vez que as ações podem não estar sendo custeadas por recursos orçamentários.

Os critérios da política de financiamento do SUS também não ficaram visíveis no mapeamento,aliás, esta é a variável que menos contribuiu para a discriminação dos municípios. Como visto, parcela dos recursos distribuídos guarda relação com o número de pessoas atendidas pelo município e o grau de complexidade do atendimento. Entretanto,volume significativo dos recursos está associado com os incentivos a ações estratégicas estabelecidas pelo Ministério da Saúde,sendo distribuído somente aos municípios que executam tais ações, como é o caso do Programa Saúde da Família.

As considerações, ora descritas, limitam-se especificamente à comparação da ti pologia da estrutura do sistema de saúde,índice de esforços em atenção básica e política de financiamento da saúde nos municípios (recursos próprios e transferências do SUS) no exercício de 200I. As variáveis representam aspectos dinâmicos da política de saúde,algumas alterando mais rapidamente e outras mais lentamente a cada ano; conseqüentemente,as categorias podem mudar de posição no mapeamento. Como base para novas pesquisas,sugere-se a comparação das variáveis em outros exercícios financeiros,com o intuito de verificar a evolução da política de financiamento dos municípios e os indicadores de esforços em saúde. 
- RELAÇÃO ENTRE GASTOS COM SAÚDE, ÍNDICE DE ESFORÇOS DA ATENÇÃO BÁSICA EM SAÚDE...• PATRÍCIA SIQUEIRA VARELA • MILTON CARLOS FARINA

\section{REFERÊNCIAS}

AFONSO,A.; AUBYN,M. St. Non-parametric approaches to education and health expenditure efficiency in OECD countries. ISEG-UTL Economics Working,paper n. I/2004/DE/CISEP/UECE, Febr. 2004.

AFONSO,A.; SCHUKNECHT,L.; TANZI,Vito. Public sector efficiency: an international comparison. ECB Working, paper series n. 242,Jul. 2003.

ARISTIGUETA,Maria P.; COOKSY,Leslie J.; Nelson,Carl W. The role of social indicators in the developing a managing for results system. Public Performance \& Management Review,v. 24,n. 3,p. 254-269,March 200I.

ARRETCHE,A. Financiamento federal e gestão local de políticas sociais: o difícil equilíbrio entre regulação,responsabilidade e autonomia. Ciência \& Saúde Coletiva,v. 8,n. 2,p. 33I-345,2003.

BARROS,E. Financiamento do sistema de saúde no Brasil: marco legal e comportamento do gasto. In: Organização Pan-Americana da Saúde. Série técnica projeto de desenvolvimento de sistemas e serviços de saúde,Brasília,2003.

BORGER,B. de; KERSTENS,K. Costefficiency of Belgian local governments: a comparative analysis of FDH,DEA and econometric approaches. Regional Science and Urban Economics,Amsterdam, v. 26,n. 2,p. 145-I70,1996.

BORGER,B. de etal. Explaining differences in productive efficiency: An application to Belgian munici palities. Public Choice,Leiden,v. 80,n. 3-4,p. 339-358,Sept. I994.

BRASIL. Constituição (I988). Constituição da República Federativa do Brasil. 21. ed. São Paulo: Saraiva,200I. (Coleção Saraiva de legislação).

Emenda Constitucional n. 29,de 14.2.2000. Legislação do SUS. Brasília: Conass,2003.

BRASIL. Ministério da Saúde. Norma Operacional Básica do Sistema Único de Saúde,5.11.1996 (NOB/SUS oI/96). Legislação do SUS. Brasília: Conass,200za.

. Norma Operacional de Assistência à Saúde. Portaria GM/MS n. 373,de 26.2.2002 -(NOAS/ SUS OI/O2). Legislação do SUS. Brasília: Conass,2003b.

BRESSER PEREIRA,L. C. A reforma do Estado dos anos 9o: lógica e mecanismos de controle. Revista Lua Nova, São Paulo,n. 45,p. 49-95,set./dez. I998.

BURKHEAD,J. Orçamento público. Rio de Janeiro: Fundação Getulio Vargas,I97I.

DATASUS. Disponível em: <http://www.datasus.gov.br>. Acesso em: 2004.

FUNDAÇÃO SEADE. Índice paulista de responsabilidade social: continuidade e desdobramentos - geração de indicador de esforços em saúde. Disponível em: <http://www.al.sp.gov/index_iprs. htm>. Acesso em: 20 set. 2003.

GUPTA,S.; VERHOEVEN,M. The efficiency of governmentexpenditure Experiences from África. Journal of Policy Modeling,New York,v. 23,n. 4,p. 433-467,200I.

HAIR JUNIOR,J. F. etal. Multivariate data analysis. 5. ed. New Jersey: Prentice-Hall,I998.

HOFFMAN,D. L.; FRANKE,G. R. Correspondence analysis: graphical representation of categorical data in marketing research. Journal of Marketing Research, Chicago,v. 23,n. 3,p. 213-227,Aug. I986.

JANNUZZI,P. M. Indicadores sociais no Brasil: conceitos,fontes de dados e aplicações. São Paulo: Alínea,200I. 
MAROCO,J. Análise estatística: com utilização do SPSS. 2. ed. Lisboa: Silabo,2003.

PESTANA,M. H.; GAGEIRO,J. N. Análise de dados para ciências sociais: a complementariedade do SPSS. 2. ed. Lisboa: Silabo,[I99-].

MENDES,Á. N.; MARQUES,R. M. Os (des)caminhos do financiamento do SUS. Saúde em debate, Rio de Janeiro,v. 27,n. 65,p. 389-404,set./dez. 2003.

SCARPIN,J. E. Estudo dos fatores condicionantes do indice de desenvolvimento humano nos municípios do Estado do Paraná: instrumento de controladoria para a tomada de decisões na gestão governamental. 2006. Dissertação (Mestrado em Controladoria e Contabilidade) -Faculdade de Economia,Administração e Contabilidade,Universidade de São Paulo,São Paulo,20o6

SCHOMMER,P. C. Gestão pública no Brasil: notícias do teatro de operações. Revista de Administração de Empresas,São Paulo,v. 43,n. 4,p. IO2-IO7,out./dez 2003.

SOUSA,M. C. S.; CRIBARI NETO,F.; STOSIC,B. Explaining DEA technical efficiency scores in an outlier corrected environment: the case of public services in Brazilian municipalities. In: ENCONTRO NACIONAL DE ECONOMIA - ENANPEC,31.,2003, Porto Seguro. Anais... Porto Seguro: Anpec,2003.

\section{TRAMITAÇÃO}

Recebido em 21/12/2005

Aprovado em 17/7/2006 
Copyright of Revista de Administração Mackenzie is the property of Universidade Presbiteriana Mackenzie, RAM-Revista de Administracao Mackenzie and its content may not be copied or emailed to multiple sites or posted to a listserv without the copyright holder's express written permission. However, users may print, download, or email articles for individual use. 